\title{
Publisher Correction: Discovery of rare variants associated with blood pressure regulation through meta-analysis of 1.3 million individuals
}

Correction to: Nature Genetics https://doi.org/10.1038/s41588-020-00713-x, published online 23 November 2020.

In the version of this article originally published, the e-mail address of corresponding author Patricia B. Munroe was incorrect. The error has been corrected in the HTML and PDF versions of the article.

Praveen Surendran, Elena V. Feofanova DD, Najim Lahrouchi, loanna Ntalla, Savita Karthikeyan, James Cook, Lingyan Chen, Borbala Mifsud, Chen Yao, Aldi T. Kraja, James H. Cartwright, Jacklyn N. Hellwege, Ayush Giri, Vinicius Tragante, Gudmar Thorleifsson, Dajiang J. Liu, Bram P. Prins, Isobel D. Stewart, Claudia P. Cabrera, James M. Eales, Artur Akbarov, Paul L. Auer, Lawrence F. Bielak, Joshua C. Bis, Vickie S. Braithwaite, Jennifer A. Brody, E. Warwick Daw, Helen R. Warren, Fotios Drenos, Sune Fallgaard Nielsen, Jessica D. Faul, Eric B. Fauman, Cristiano Fava, Teresa Ferreira,

Christopher N. Foley, Nora Franceschini, He Gao, Olga Giannakopoulou, Franco Giulianini, Daniel F. Gudbjartsson, Xiuqing Guo, Sarah E. Harris, Aki S. Havulinna, Anna Helgadottir, Jennifer E. Huffman, Shih-Jen Hwang, Stavroula Kanoni, Jukka Kontto, Martin G. Larson, Ruifang Li-Gao, Jaana Lindström, Luca A. Lotta, Yingchang Lu, Jian'an Luan, Anubha Mahajan, Giovanni Malerba, Nicholas G. D. Masca, Hao Mei, Cristina Menni, Dennis O. Mook-Kanamori, David Mosen-Ansorena, Martina Müller-Nurasyid, Guillaume Paré, Dirk S. Paul, Markus Perola, Alaitz Poveda, Rainer Rauramaa, Melissa Richard, Tom G. Richardson, Nuno Sepúlveda, Xueling Sim, Albert V. Smith, Jennifer A. Smith, James R. Staley, Alena Stanáková, Patrick Sulem, Sébastien Thériault, Unnur Thorsteinsdottir, Stella Trompet, Tibor V. Varga, Digna R. Velez Edwards, Giovanni Veronesi, Stefan Weiss, Sara M. Willems, Jie Yao, Robin Young, Bing Yu, Weihua Zhang, Jing-Hua Zhao, Wei Zhao, Wei Zhao, Evangelos Evangelou, Stefanie Aeschbacher, Eralda Asllanaj, Stefan Blankenberg, Lori L. Bonnycastle, Jette Bork-Jensen, Ivan Brandslund, Peter S. Braund, Stephen Burgess, Kelly Cho, Cramer Christensen, John Connell, Renée de Mutsert, Anna F. Dominiczak, Marcus Dörr, Gudny Eiriksdottir, Aliki-Eleni Farmaki, J. Michael Gaziano, Niels Grarup, Megan L. Grove, Göran Hallmans, Torben Hansen, Christian T. Have, Gerardo Heiss, Marit E. Jørgensen, Pekka Jousilahti, Eero Kajantie, Mihir Kamat, AnneMari Käräjämäki, Fredrik Karpe, Heikki A. Koistinen, Csaba P. Kovesdy, Kari Kuulasmaa, Tiina Laatikainen, Lars Lannfelt, I-Te Lee, Wen-Jane Lee, LifeLines Cohort Study*, Allan Linneberg, Lisa W. Martin, Marie Moitry, Girish Nadkarni, Matt J. Neville, Colin N. A. Palmer, George J. Papanicolaou, Oluf Pedersen, James Peters, Neil Poulter, Asif Rasheed, Katrine L. Rasmussen,

N. William Rayner, Reedik Mägi, Frida Renström, Rainer Rettig, Jacques Rossouw, Pamela J. Schreiner, Peter S. Sever, Emil L. Sigurdsson, Tea Skaaby, Yan V. Sun, Johan Sundstrom, Gudmundur Thorgeirsson, Tõnu Esko, Elisabetta Trabetti, Philip S. Tsao, Tiinamaija Tuomi, Stephen T. Turner, Ioanna Tzoulaki, Ilonca Vaartjes, Anne-Claire Vergnaud, Cristen J. Willer, Peter W. F. Wilson, Daniel R. Witte, Ekaterina Yonova-Doing, He Zhang, Naheed Aliya, Peter Almgren, Philippe Amouyel, Folkert W. Asselbergs, Michael R. Barnes, Alexandra I. Blakemore, Michael Boehnke, Michiel L. Bots, Erwin P. Bottinger, Julie E. Buring, John C. Chambers, Yii-Der Ida Chen, Rajiv Chowdhury, David Conen, Adolfo Correa, George Davey Smith, Rudolf A. de Boer, Ian J. Deary, George Dedoussis, Panos Deloukas, Emanuele Di Angelantonio, Paul Elliott, EPIC-CVD*, EPIC-InterAct*, Stephan B. Felix, Jean Ferrières, lan Ford, Myriam Fornage, Paul W. Franks, Stephen Franks, Philippe Frossard, Giovanni Gambaro, Tom R. Gaunt, Leif Groop, Vilmundur Gudnason, Tamara B. Harris, Caroline Hayward, Branwen J. Hennig, Karl-Heinz Herzig, Erik Ingelsson, Jaakko Tuomilehto, Marjo-Riitta Järvelin, J. Wouter Jukema, Sharon L. R. Kardia, Frank Kee, Jaspal S. Kooner, Charles Kooperberg, Lenore J. Launer, Lars Lind, Ruth J. F. Loos, Abdulla al Shafi. Majumder, Markku Laakso, Mark I. McCarthy, Olle Melander, Karen L. Mohlke, Alison D. Murray, Børge Grønne Nordestgaard, Marju Orho-Melander, Chris J. Packard, Sandosh Padmanabhan, Walter Palmas, Ozren Polasek, David J. Porteous, Andrew M. Prentice, Michael A. Province, Caroline L. Relton, Kenneth Rice, Paul M. Ridker, Olov Rolandsson, Frits R. Rosendaal, Jerome I. Rotter, Igor Rudan, Veikko Salomaa, Nilesh J. Samani, Naveed Sattar, Wayne H.-H. Sheu, Blair H. Smith, Nicole Soranzo, Timothy D. Spector, John M. Starr, Sylvain Sebert, Kent D. Taylor, Timo A. Lakka, Nicholas J. Timpson, Martin D. Tobin, Understanding Society Scientific Group*, Pim van der Harst, Peter van der Meer, Vasan S. Ramachandran, Niek Verweij, Jarmo Virtamo, Uwe Völker, David R. Weir, Eleftheria Zeggini, Fadi J. Charchar, Million Veteran Program, Nicholas J. Wareham, Claudia Langenberg, Maciej Tomaszewski, Adam S. Butterworth, Mark J. Caulfield, John Danesh, Todd L. Edwards, Hilma Holm, Adriana M. Hung, Cecilia M. Lindgren, Chunyu Liu, Alisa K. Manning, Andrew P. Morris, Alanna C. Morrison, Christopher J. O'Donnell, Bruce M. Psaty, Danish Saleheen, Kari Stefansson, Eric Boerwinkle, Daniel I. Chasman (D, Daniel Levy, Christopher Newton-Cheh, Patricia B. Munroe (D) and Joanna M. M. Howson (D)

${ }^{\star}$ Lists of authors and their affiliations appear online.

Published online: 16 March 2021 